\title{
A methylated oligonucleotide inhibits IGF2 expression and enhances survival in a model of hepatocellular carcinoma
}

\author{
Xiaoming Yao, ${ }^{1} \mathrm{Ji}-\mathrm{Fan} \mathrm{Hu},{ }^{2}$ Mark Daniels, ${ }^{1}$ Hadas Shiran, ${ }^{2}$ Xiangjun Zhou, ${ }^{2}$ Huifan Yan, ${ }^{3}$ \\ Hongqi Lu, ${ }^{3}$ Zhilan Zeng, ${ }^{1}$ Qingxue Wang, ${ }^{1}$ Tao Li, ${ }^{1}$ and Andrew R. Hoffman ${ }^{1}$ \\ ${ }^{1}$ Medical Service, Veterans Affairs Palo Alto Health Care System, and Division of Endocrinology, Department of Medicine, \\ Stanford University, Palo Alto, California, USA \\ ${ }^{2}$ GMR Epigenetics Corporation, Sunnyvale, California, USA \\ ${ }^{3}$ Department of Pharmacology, Shanghai Institute of Pharmaceutical Industry, Shanghai, People's Republic of China
}

\begin{abstract}
IGF-II is a mitogenic peptide that has been implicated in hepatocellular oncogenesis. Since the silencing of gene expression is frequently associated with cytosine methylation at cytosine-guanine $(\mathrm{CPG})$ dinucleotides, we designed a methylated oligonucleotide (MON1) complementary to a region encompassing IGF2 promoter $\mathrm{P} 4$ in an attempt to induce DNA methylation at that locus and diminish IGF2 mRNA levels. MON1 specifically inhibited IGF2 mRNA accumulation in vitro, whereas an oligonucleotide (ON1) with the same sequence but with nonmethylated cytosines had no effect on IGF2 mRNA abundance. MON1 treatment led to the specific induction of de novo DNA methylation in the region of IGF2 promoter hP4. Cells from a human hepatocellular carcinoma (HCC) cell line, Hep 3B, were implanted into the livers of nude mice, resulting in the growth of large tumors. Animals treated with MON1 had markedly prolonged survival as compared with those animals treated with saline or a truncated methylated oligonucleotide that did not alter IGF2 mRNA levels in vitro. This study demonstrates that a methylated sense oligonucleotide can be used to induce epigenetic changes in the IGF2 gene and that inhibition of IGF2 mRNA accumulation may lead to enhanced survival in a model of HCC.
\end{abstract}

J. Clin. Invest. 111:265-273 (2003). doi:10.1172/JCI200315109.

\section{Introduction}

IGF-II, a mitogenic peptide that has been implicated in oncogenesis, exerts its hormonal, paracrine, and autocrine bioactivity through its interaction with the type I IGF receptor. The peptide is inactivated by the mannose6-phosphate receptor/IGF2 receptor (M6P/IGF2R), which binds IGF-II on the cell surface and transports it to the lysosome for degradation (1). The gene encoding IGF-II (IGF2) is overexpressed in a wide variety of neoplasms, including hepatocellular carcinoma (HCC) (2), and it is thought that the gene may serve as an autocrine tumor enhancer. The type I IGF receptor is found in many HCCs and cell lines, providing a means for IGF-II to exert its mitogenic activity (3). Moreover, M6P/IGF2R is mutated in the majority of dysplastic liver lesions and HCC. It has been postulated that inactivation of a single

Received for publication January 22, 2002, and accepted in revised form October 16, 2002.

Address correspondence to: Andrew R. Hoffman, Building 101, Room B2-125, Veterans Affairs Palo Alto Health Care System, 3801 Miranda Avenue, Palo Alto, California 94304, USA. Fax: (650) 856-8054; Phone: (650) 725-2908;

E-mail: arhoffman@stanford.edu.

Conflict of interest: The authors have declared that no conflict of interest exists.

Nonstandard abbreviations used: methylated oligonucleotide 1 (MON1); oligonucleotide 1 (ON1); hepatocellular carcinoma (HCC); mannose-6-phosphate receptor/IGF2 receptor (M6P/IGF2R); cytosine-guanine (CpG); $5^{\prime}$-methylcytosine $\left(\mathrm{m}^{5} \mathrm{C}\right)$; DNA methyltransferase-1 (DNMT1); deoxy-cytosinetriphosphate (dCTP); dATP + dCTP + dTTP + dGTP (dNTP); control oligonucleotide (CON); total nucleic acid (TNA); $\alpha$-fetoprotein (AFP).
$M 6 P / I G F 2 R$ allele leads to a decreased number of IGF-II receptors, and as a result there is diminished IGF-II degradation, increased IGF-II levels, and ultimately clonal expansion of premalignant hepatocytes (4). Upregulation of IGF2 and downregulation of M6P/IGF2R would work synergistically to increase tumor growth.

Using microarray technology to determine which genes are overexpressed in neoplasms, investigators showed that two IGF2 transcripts were upregulated to a far greater extent than were any other mRNA transcripts in colorectal cancer (5). IGF2 expression is also upregulated in the liver tumors that develop in mice transgenic for the hepatitis B virus (HBV) (6), and we have shown that hepatic IGF2 mRNA abundance varies with $\mathrm{HBV}$ expression in a coordinated fashion in these transgenic mice (7). Similar findings have been reported in human HCC, where IGF2 expression is highly correlated with the production of the HBV X gene product (8). Moreover, hepatic malignancies frequently develop in mice that carry the Igf 2 transgene (9). $\beta$-cell oncogenesis was markedly inhibited in Igf2 knockout transgenic mice (10). In a SV40 transgenic model, animals carrying the mutated Igf2 allele developed fewer and smaller liver tumors than those carrying the normal Igf2 allele (11). Thus, IGF2 appears to be a second "survival" signal for oncogene-induced abnormal cell growth in a variety of tumors (12).

Since IGF2 overexpression is so closely linked to hepatic oncogenesis, strategies designed to inhibit IGF-II synthesis might lead to prevention or amelioration of HCC. 
The silencing of genes during development is frequently associated with the methylation of cytosine-guanine (CpG) islands (13). Inhibitors of DNA methylation may lead to the reexpression of genes that are silenced in cancer (14). We have previously shown that 5 -azacytidine, an inhibitor of DNA methylation, specifically increases IGF2 synthesis from human promoter $\mathrm{P} 4$ and from the cognate mouse promoter P3 (15). We therefore devised a strategy to methylate regions near IGF2 promoters in order to decrease IGF2 synthesis in HCC and thereby diminish tumor growth.

Therapeutic oligonucleotides are thought to inhibit gene expression by preventing translation through steric hindrance at the ribosome or by annealing to mRNA and forming double-stranded RNA that is subject to rapid degradation by intracellular RNases (16). In creating a methylated sense oligonucleotide, we propose a novel mechanism whereby oligonucleotide therapy may work: the induction of site-specific DNA methylation. This technique utilizes a synthetic oligonucleotide in which the cytosine residues in the $5^{\prime}$-CpG-3' dinucleotides are replaced by 5 '-methylcytosine $\left(\mathrm{m}^{5} \mathrm{C}\right)$. We hypothesize that binding of the synthetic $m^{5} \mathrm{CpG}$ probe to one strand of the gene forms a hemimethylated DNA intermediate, which has a "replication fork"-like structure and is thus a preferred substrate of DNA methyltransferase-1 (DNMT1) (17). DNMT1 would catalyze DNA methylation of the first strand of the intermediate. When the synthetic probe leaves that locus, the methylated strand would reanneal to its complementary unmethylated DNA strand to form a second hemimethylated substrate for DNMT1, and DNMT1 would immediately methylate the second strand of the gene. As a result, the gene would become fully methylated at the targeted site. Cellular transcription suppressors, like MeCP2 (18) and other proteins, could then presumably bind to the methylated DNA region and block the expression of the targeted gene. In this study, we utilize a methylated oligonucleotide that is complementary to a region encompassing the human IGF2 promoter P4 to inhibit IGF2 synthesis and to examine its effect on the growth of HCC in a mouse model.

\section{Methods}

Materials. Human HCC cell lines Hep 3B and Hep G2 were purchased from American Tissue Culture Collection (Manassas, Virginia, USA). Human embryonic fibroblasts were cultured from human embryonic skin in our laboratory as previously described $(15,19)$. Male $\mathrm{Balb} / \mathrm{c}$ athymic (nu/nu) nude mice were purchased from Simonsen Laboratory (Gilroy, California, USA).

We designed phosphorothioate oligonucleotides in which the cytosines in $\mathrm{CPG}$ dinucleotides are replaced by $\mathrm{m}^{5} \mathrm{CpG}$. The oligonucleotides were purchased from the Stanford University facility and from Proligo LLC (Boulder, Colorado, USA). Methylated oligonucleotide 1 (MON1: AGC C ${ }^{\mathrm{m}} \mathrm{CG} \mathrm{GG}^{\mathrm{m}} \mathrm{C}$ TGG GAG GAG T ${ }^{\mathrm{m}} \mathrm{CG} \mathrm{G}$ ) is a 22-mer sense oligonucleotide that is complementary to a region of human IGF2 promoter P4 (GenBank accession no. AC006408) and mouse Igf2 promoter P3. MON2 is a $19-$ mer $\left(\mathrm{C}^{\mathrm{m}} \mathrm{CG} \mathrm{GG}{ }^{\mathrm{m}} \mathrm{C}\right.$ TGG GAG GAG Tm $\mathrm{CG}$ G) that is identical to MON1, except that the first three nucleotides have been truncated. CON1 is a control cognate of the MON1 oligonucleotide, consisting of a methylated 22-mer oligonucleotide with a random sequence ( $\mathrm{TGC}^{\mathrm{m}} \mathrm{CGG} A \mathrm{~A}^{\mathrm{m}} \mathrm{C}$ GTG AGC ACT T ${ }^{\mathrm{m}} \mathrm{CT} \mathrm{G}$ ). As an oligonucleotide control, CON1 is not related to IGF2, but it contains the same number of $\mathrm{m}^{5} \mathrm{C}$ residues as MON1. Another two control oligonucleotides, ON1 and ON2, are identical to MON1 and MON2 in sequence, except that they contain unmethylated cytosine residues. The oligonucleotides were purified by high-performance liquid chromatography yielding a purity of more than $95 \%$ of full-length oligonucleotides. Cell culture. Hep 3B and Hep G2 cells were maintained in MEM medium (GIBCO BRL, Carlsbad, California, USA) supplemented with $10 \%$ FBS, $200 \mathrm{mM}$ glutamine, $100 \mathrm{mM}$ MEM sodium pyruvate, and MEM nonessential amino acids at $37^{\circ} \mathrm{C}$ with $5 \% \mathrm{CO}_{2}$. Tumor cells were seeded in six-well plates at a density of $2 \times 10^{5}$ cells $/ \mathrm{ml}$ and were treated with various concentrations of liposome-encapsulated MON1, MON2, or CON in $1 \mathrm{ml}$ of fresh media. Polynucleotide oligonucleotides were delivered into cells with GenePorter Transfection Reagent (Gene Therapy Systems, San Diego, California, USA). After 24 hours of treatment, $1 \mathrm{ml}$ of fresh media was added, and the cells were allowed to grow until they became confluent for RNA analysis. Without liposome delivery, a dose of the oligonucleotide that was higher by an order of magnitude was needed to achieve the same inhibiting efficiency.

Cellular distribution of MON1. MON1 was 5'-end labeled with fluorescent dye (FluoReporter, Molecular Probes, Eugene, Oregon, USA), according to the protocol provided by the manufacturer. Hep 3B cells $\left(2 \times 10^{4}\right.$ cells $/ \mathrm{ml}$ ) were seeded in a glass chamber and treated with fluorescently labeled MON1. After overnight incubation, cells were washed three times with PBS, covered with a slide, and examined for fluorescent signals under a confocal microscope.

IGF2 mRNA quantitation. Confluent cells from treatment and control wells were directly lysed with $0.6 \mathrm{ml}$ of solution $\mathrm{D}$ (4 M guanidium thiocyanate solution containing $1 \% \beta$-mercaptoethanol, $2.5 \mathrm{mM}$ sodium citrate, and $0.5 \%$ sarcosyl). Total nucleic acid (TNA) was extracted and purified using our previously described method $(15,20)$. TNA samples were directly used for cDNA synthesis with RNA reverse transcriptase. Because TNA samples contain both genomic DNA and synthesized cDNA, we named it cTNA to distinguish cDNA synthesized by common procedures. The major advantage of cTNA over CDNA is that the same pair of PCR primers will amplify both genomic DNA and mRNA at the same time. The amplified genomic DNA can be used as a reliable internal control. As a result, the expression of IGF2 can be quantitated as a function of total genomic DNA, in addition to $\beta$-actin or other control genes. 
IGF2 expression after methylated oligonucleotide treatment was first examined by PCR amplification. Briefly, cDNA samples were amplified in a 3.0- $\mu$ l reaction mixture in the presence of $50 \mu \mathrm{M} \mathrm{dATP}+\mathrm{dCTP}+$ $\mathrm{dTTP}+\mathrm{dGTP}(\mathrm{dNTP}), 0.2 \mu \mathrm{M}$ IGF2 primers, $0.25 \mu \mathrm{Ci}$ $\left[\alpha-{ }^{32} \mathrm{P}\right]$ deoxy-cytosine-triphosphate (dCTP) (Amersham Co., Arlington Heights, Illinois, USA), and 0.125 U KlenTaq1 DNA polymerase (Ab Peptides Inc., St. Louis, Missouri, USA). DNA was amplified for 32 cycles at $94^{\circ} \mathrm{C}$ for 15 seconds and $65^{\circ} \mathrm{C}$ for 40 seconds, followed by a 30 -second extension at $72^{\circ} \mathrm{C}$. The PCR products were separated on $5 \%$ polyacrylamide-urea gel and scanned for quantitation by a PhosphoImager Analyzer (Molecular Dynamics, Sunnyvale, California, USA).

The oligonucleotide primers used for quantitating IGF2 expression were number 3038 (5' -primer, TGGCCCTCCTGGAGACGTACTGTGC) and number 2384 (3'primer, TTGGAAGAACTTGCCCACGGGGTATC). This pair of IGF2 primers crosses IGF2 intron 8 such that genomic DNA and mRNA give PCR products of various sizes that can be easily separated on $5 \%$ polyacrylamide-urea gel for $I G F 2$ quantitation.

IGF2 expression in Hep 3B cells treated with MON1 was further quantitated using a Northern hybridization method $(7,21)$. An IGF2 probe that covers partial sequencing of exons 7, 8, and 9 (GenBank accession no. J03242: 1016-1351) was prepared by PCR primers 995 (forward, 5'-GTCCCCTGATTGCTCTACCCAC) and 996 (reverse, 5' GAGGGTATGTGAAGGGTGTTTAAAGC) and was cloned into a TA Vector (Invitrogen, Carlsbad, California, USA). Probe DNA was labeled by a singleprimer PCR method. Specifically, probe labeling was carried out in a $30-\mu \mathrm{l}$ reaction mixture containing 10 ng of probe, $2 \mathrm{U}$ of KlenTaq1, $2 \mu \mathrm{l}$ of $3 \mu \mathrm{M}$ primer 996, and $25 \mu \mathrm{Ci}$ of $\left[\alpha^{32} \mathrm{P}\right] \mathrm{dCTP}$. The PCR reaction used for labeling the probe was set as $95^{\circ} \mathrm{C}$ for 2 minutes, followed by 35 cycles of amplification at $95^{\circ} \mathrm{C}$ for $20 \mathrm{sec}$ onds, $65^{\circ} \mathrm{C}$ for 30 seconds, and $72^{\circ} \mathrm{C}$ for 1 minute. The labeled probe was purified by a DNA purification column (Princeton Separations Inc., Adelphia, New Jersey, USA) and used for Northern hybridization as previously described $(7,21)$.

Measurement of DNA methylation. Genomic DNA was isolated and purified using the method described previously (22). DNA samples (20 $\mu \mathrm{g})$ extracted from Hep $3 \mathrm{~B}$ cells were fragmented with PvuII and then digested with the DNA methylation-sensitive restriction enzyme HpaII. Methylated genomic DNA resists the digestion of HpaII and yields a full-length band in agarose gel. The unmethylated DNA, in contrast, is digested by the restriction enzyme and produces small fragments in the gel. For a control, genomic DNA was also digested with the DNA methylation-insensitive restriction enzyme $M s p I$, which cuts genomic DNA whether it is methylated or unmethylated.

The status of DNA methylation in Hep 3B cells was determined by Southern blotting (22). Briefly, HpaIIdigested DNA was separated on $1.2 \%$ agarose gel and transferred onto Hydrobond-N filter (Amersham Life
Science, Arlington Heights, Illinois, USA). The filter was hybridized with $\left[\alpha-{ }^{32} \mathrm{P}\right] \mathrm{dCTP}-$ labeled DNA probe prepared from the most proximal promoter of the human IGF2 gene. After washing, the filter was scanned by a PhosphoImager Scanner (Molecular Dynamics).

DNA methylation induced by methylated oligonucleotide was also measured by the bisulfite/sequencing method as described by Frommer et al. (23) with some modifications (24). In brief, genomic DNAs were treated with freshly prepared $3.5 \mathrm{M} \mathrm{NaHSO}_{3}$ containing $1 \mathrm{mM}$ hydroquinone ( $\mathrm{pH}$ 5.0) and purified with a DNA purification kit (QIAGEN Inc., Chatsworth, California, USA). DNA samples were diluted and amplified with PCR amplification using conditions as described above. Primers used for PCR amplification include 491 (5' -primer, GGATATTGAGGATTGGTTT[T/C]GGGGTT) and 494 (3'-primer, CAACCTTCAAAAAAAATATCCTCTTCAAT) or 1232 (5' primer: TAAAAAACCCATAACACTTCGCTACG) and 494. The status of DNA methylation in various regions was examined with a Sequenase 2.0 sequencing kit (United States Biochemical, Cleveland, Ohio, USA) and analyzed with a PhosphoImager Analyzer.

Tumor study. Male Balb/c athymic (nu/nu) nude mice, 4-5 weeks old and weighing 20-25 g, were kept under pathogen-free conditions, fed standard food, and given free access to sterilized water. Housing and all procedures followed protocols approved by the Veterans Affairs Palo Alto Health Care System Research Service.

Mice were anesthetized intraperitoneally with 80 $\mathrm{mg} / \mathrm{kg}$ of ketamine and $10 \mathrm{mg} / \mathrm{kg}$ of xylazine. The mice were placed in a supine position, and a transection incision below the sternum was made. Hep 3B cells $\left(2 \times 10^{6}\right)$ were slowly injected into the upper left lobe of the liver with a 28-gauge needle. The abdomen was then closed with 6-0 silk suture.

In one animal study, MON1 at two doses $(0.7 \mathrm{mg} / \mathrm{kg}$ and $7 \mathrm{mg} / \mathrm{kg}$ ) was administered through the tail vein 10 days after Hep 3B cell implantation. In another separate animal study, 4 weeks after tumor cell injection, the mice were randomly divided into treatment groups to receive oligonucleotide or saline infusions. MON1 and MON2 oligonucleotides were given intravenously at a dose of $10 \mathrm{mg} / \mathrm{kg}$ from the tail vein every 2 days. Since we lack a reliable liposomal delivery system for our in vivo studies, we have employed naked oligonucleotides for our tumor studies in animals. Tissues were collected after death and stored at $-80^{\circ} \mathrm{C}$ until analysis. At the end of the study (20 weeks), the remaining surviving mice were sacrificed after ketamine anesthesia.

\section{Results}

Inbibition of IGF2 in vitro. We first sought to determine if the methylated oligonucleotide would inhibit IGF2 gene expression in vitro. We exposed cultured normal human fibroblasts to a low dose $(2 \mu \mathrm{M})$ of the methylated 22-mer oligonucleotide MON1. Human fibroblasts (HSK 09) were cultured from fresh embryonic tissues that contain a relatively high abundance of IGF2. 


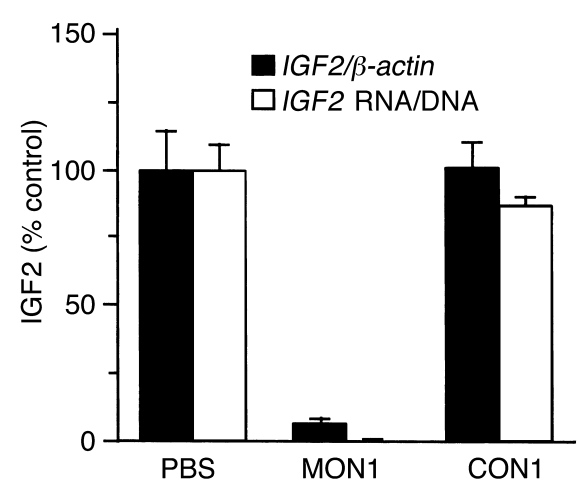

Figure 1

IGF2 expression in human HSK 09 cells treated with the methylated oligonucleotide MON1, as assessed by RT-PCR. IGF2 mRNA abundance was normalized by comparing its density with that of either IGF2 genomic DNA or an internal control, $\beta$-actin.

After low-dose MON1 treatment, IGF2 mRNA expression decreased by more than $95 \%$. Incubation with a nonspecific oligonucleotide control (CON1), however, did not alter IGF2 expression (Figure 1). Similar results were also obtained when MON1 was tested in a second human fibroblast cell line, HSK 06 (data not shown).

In order to learn if the oligonucleotide would have a similar effect in malignant cells, two human liver cancer cell lines, Hep 3B and Hep G2, were used to examine the inhibitory effect of MON1. Although both lines are derived from hepatic cancers, they differ in that the Hep $3 \mathrm{~B}$ cell is positive for HBV, whereas Hep G2 is negative for HBV. Both tumor cell lines expressed very high levels of IGF2 mRNA. After incubation with MON1 $(2 \mu \mathrm{M})$ for 24 hours, IGF2 mRNA abundance was dramatically reduced in each of the tumor lines (Figure 2a). This inhibition was dose dependent, with approximately 50\% inhibition of IGF2 mRNA accumulation seen after using $0.5 \mu \mathrm{M}$ MON1 (Figure 2b). The nonspecific oligonucleotide control CON1 had no significant effect on IGF2 expression (Figure 2a). Reduction of IGF2 mRNA abundance after MON1 treatment in Hep 3B cells was also confirmed with Northern blotting using a probe covering exons 7,8 , and 9 (Figure $2 \mathrm{c}$ ).

We also performed a time-course experiment on IGF2 expression. Hep $3 \mathrm{~B}$ cells were incubated with $3 \mu \mathrm{M}$ of MON1 for varying periods of time ( 8 hours, 12 hours, 24 hours, 48 hours, and 72 hours). It is interesting to note that MON1 was able to block IGF2 expression in Hep 3B cells as early as eight hours after treatment (data not shown). We then used a multiplex PCR to amplify IGF2 mRNA in order to examine the promoter-specific transcripts of IGF2 in MON1-treated cells. We found that IGF2 mRNA transcripts derived from the target hP4 promoter, as well as the other three upstream promoters were downregulated by MON1 (data not shown).

In a separate study, we examined how long the inhibition of MON1 on IGF2 expression could be maintained. We incubated Hep 3B cells with MON1 for 48 hours (passage 1). Cells were collected and then seeded in a new plate without further exposure to MON1 (passage 2). We repeated this experiment until cell passage 5 . We found that the inhibiting effect of MON1 was completely maintained in passage 2 and then lost with subsequent cell passages (data not shown). These data demonstrate that MON1-induced methylation of a

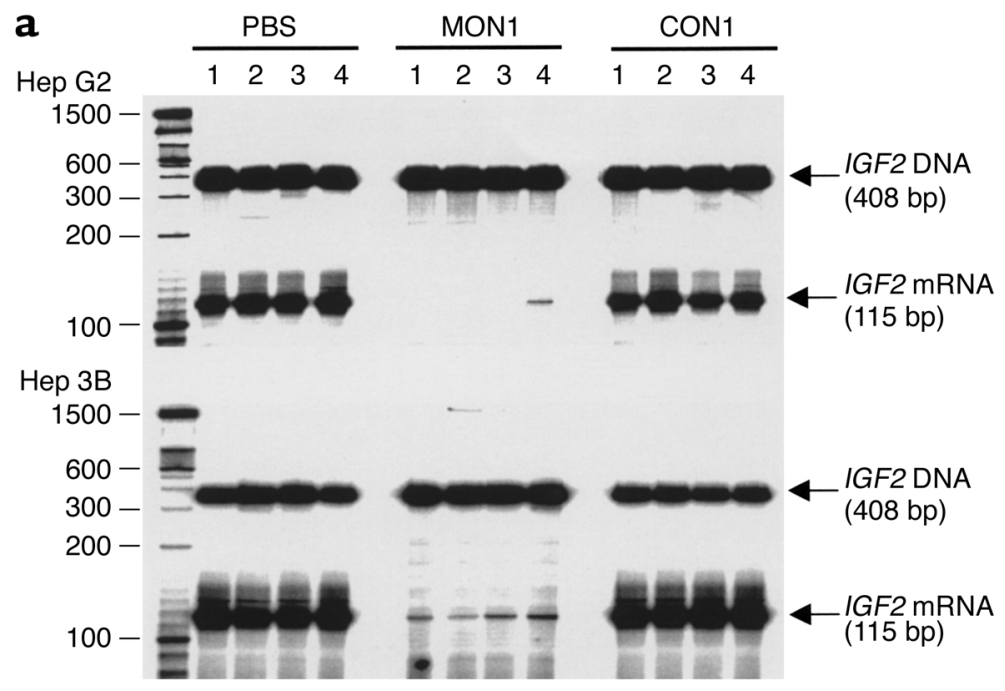

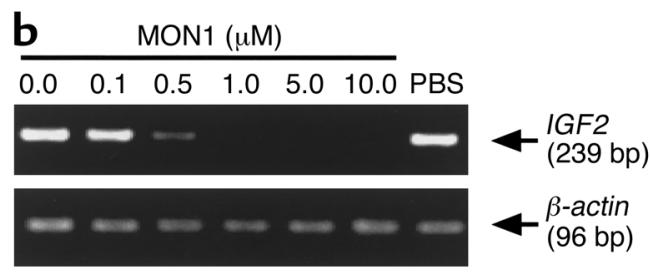

c

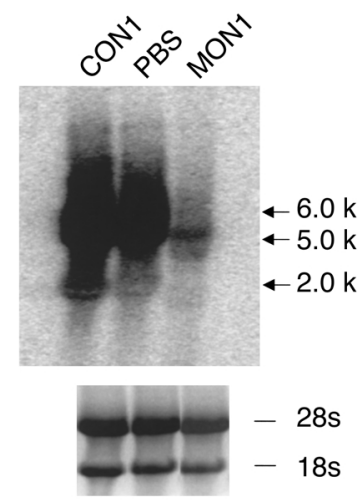

Figure 2

Inhibition of IGF2 expression by MON1 oligonucleotide in liver cancer cells. (a) Hep 3B and Hep G2 cells were seeded in six-well plates and were treated with MON1 $(2 \mu \mathrm{M})$ in a low-serum medium. As described in Methods, both IGF2 DNA and IGF2 mRNA were coamplified by the same primer set that encompasses intron 8 of the human IGF2 gene. (b) Hep 3B cells were treated with varying doses (0.0-10.0 $\mu \mathrm{M})$ of MON1 for 60 hours, and IGF2 mRNA abundance was quantitated by RT-PCR. (c) Northern blot analysis of /GF2 mRNA in Hep 3B cells after MON1 treatment using a probe covering IGF2 exons 7,8 , and 9. 


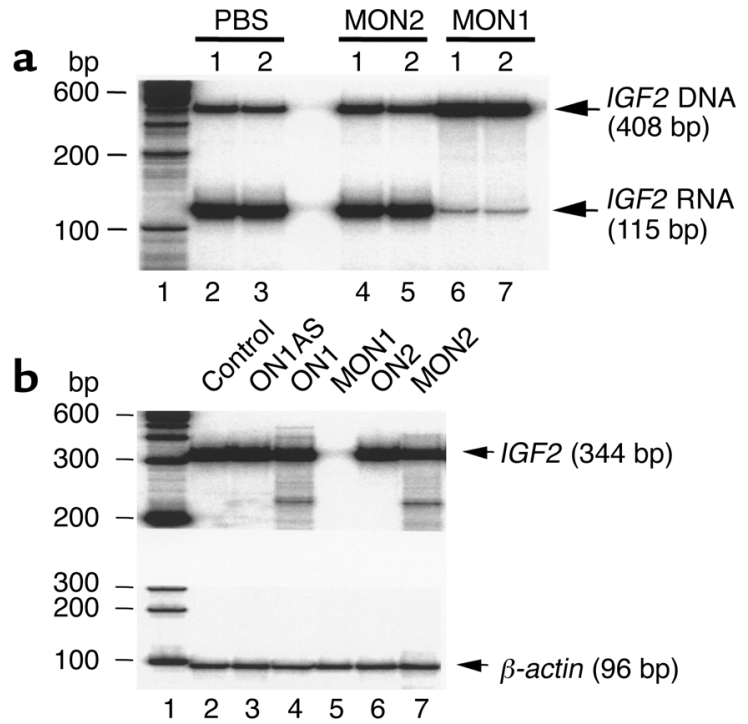

Figure 3

Specific inhibition of IGF2 expression with the methylated 22-mer oligonucleotide MON1. (a) Hep 3B cells were treated with the methylated 22-mer MON1 and the truncated methylated 19-mer MON2. The abundance of IGF2 mRNA was quantitated by RT-PCR in duplicate samples. Controls were incubated with PBS alone. (b) Hep 3B cells were treated with methylated and unmethylated oligonucleotides. Lane 1 shows a 100-bp marker; lane 2, PBS control; lane 3 , unmethylated antisense of 22-mer ON1; lane 4, unmethylated 22-mer ON1; lane 5, methylated MON1; lane 6, unmethylated 19-mer ON2; and lane 7, methylated 19-mer MON2.

IGF2 can be maintained for one more passage and is then reversed, probably by an uncharacterized DNA demethylating process.

As a further test of the specificity of the methylated oligonucleotide, we synthesized a shorter version of MON1 to determine its bioactivity. MON1 is a 22-base oligonucleotide that contains a $\mathrm{CPG}$ dinucleotide near each end of the sequence. Using the Hep $3 \mathrm{~B}$ cell line as a model, we found that truncating the original 22-mer to a 19-mer (MON2) by omitting three bases adjacent to one of the CPG sites greatly diminished the IGF2 suppressor activity of the oligonucleotide (Figure 3 ). Further reduction of the oligonucleotide length also led to oligomers that were not able to block IGF2 expression (data not shown). Finally, we exposed cells to ON1, a 22-mer oligonucleotide identical to MON1 except that all of the CpGs were unmethylated. In contrast to MON1, ON1 did not alter IGF2 mRNA abundance (Figure 3b, lane 4), demonstrating the importance of the methylated CpGs for the inhibitory action of the oligonucleotide. The inhibition of IGF2 mRNA expression was not observed using other unmethylated oligonucleotides, including ON2 (unmethylated MON2) (Figure 3b, lane 6) and ON1AS (unmethylated MON1 antisense oligonucleotide) (Figure $3 \mathrm{~b}$, lane 2 ). The expression of $\beta$-actin did not change after incubation with any of the oligonucleotides, demonstrating that the inhibition by the 22-mer methylated oligonucleotide directed against promoter P4 of the IGF2 gene was specific for $I G F 2$.
DNA methylation after treatment with the methylated oligonucleotide. We hypothesized that treatment with MON1 would lead to increased methylation of the P4 promoter and thus lead to inhibition of gene transcription. In order to induce DNA methylation, MON1 must first enter the cell and then enter the nucleus. To examine cellular localization, MON1 was fluorescently labeled and then incubated with the two human liver cell lines (Hep 3B and Hep G2). After incubation, tumor cells were washed with PBS and fixed with absolute methanol, and the cellular distribution of MON1 was examined by confocal microscopy. MON1 entered the tumor cells and was more concentrated in the nucleus than in the cytoplasm (Figure 4).

In order to determine the status of DNA methylation in Hep 3B tumor cells, IGF2 methylation was analyzed by Southern hybridization (22). Genomic DNA was first digested with the methylation-sensitive restriction enzyme HpaII, which specifically digests unmethylated CCpGG sites. Methylated DNA is resistant to HpaII and thus appears as full-length or larger fragments, whereas unmethylated DNA is subject to HpaII digestion and appears as shorter fragments on agarose gel electrophoresis. As previously described (25), genomic DNA from PBS-treated Hep 3B cells showed an absence of methylation at IGF2 promoter P4, which is consistent with the abundant IGF2 expression in these tumor cells. After the cells were incubated with MON1, a hypermethylated DNA pattern was observed in the target region (Figure 5), indicating that the specific methylated oligonucleotide induces de novo DNA methylation in the targeted DNA region.

The status of DNA methylation in the hP4 region was further delineated by a sodium bisulfite-sequencing method. After sodium bisulfite treatment, unmethylated cytosine residues are converted into uracil residues and were amplified as thymidines in the PCR reaction, whereas methylated cytosine residues remained intact. After MON1 treatment, several $\mathrm{CpG}$ dinucleotides in the hP4 region now become methylated (Figure 6). In a time-course study, we found that this de novo DNA methylation occurred in Hep 3B cells as early as 8 hours after MON1 treatment (data not shown).
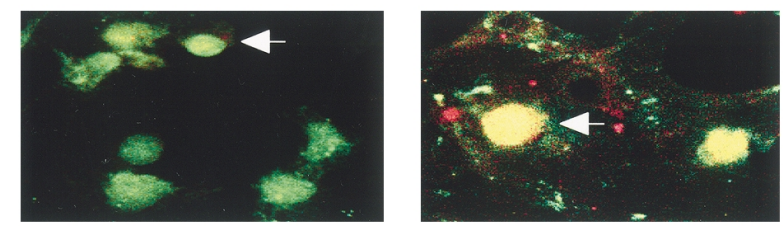

\section{Figure 4}

Nucleic enrichment of MON1 in Hep 3B tumor cells. MON1 was labeled using a fluorescence kit and was incubated with Hep 3B cells in the liposome-encapsulated form. After 24 hours of incubation, tumor cells were washed with PBS and examined by confocal microscopy. The arrows point to nuclei concentrating the fluorescent MON1 (oil, x400). Images were acquired from two separate fields with FITC (left) and combined (right) settings, respectively. 
a

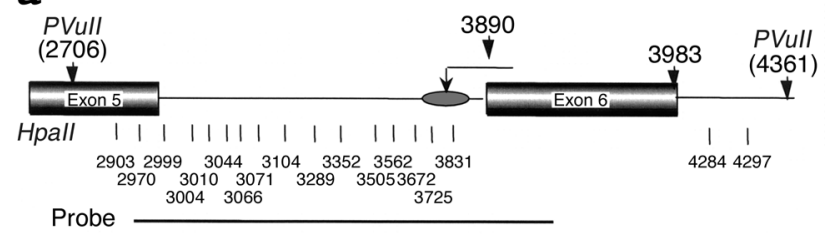

b

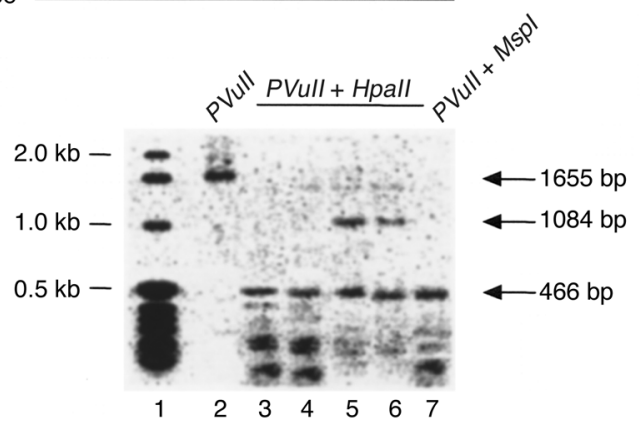

We also used sodium bisulfite sequencing to map DNA methylation patterns in IGF2 hP3 and in the H19 promoter, which are upstream and downstream of hP4, respectively. We did not see any significant changes in DNA methylation in these two regions after MON1 treatment, demonstrating that the methylation induced by MON1 is site and sequence specific (data not shown).

Effect of MON1 on survival from HCC. Having demonstrated that MON1 inhibited IGF2 mRNA levels in HCC cells in vitro, we examined whether MON1 could alter survival in a mouse model of HCC. After Hep 3B cell implantation, tumors are formed in the liver of nude mice and synthesize very high levels of the growth factor IGF2 (unpublished data). These tumors will continue to grow and eventually kill the mice. Four weeks after Hep 3B implantation, nude mice were randomly assigned to receive various treatments: saline control, the 19-mer oligonucleotide control MON2, and the 22-mer

\section{Figure 6}

DNA methylation at specific $\mathrm{CpG}$ dinucleotides in the hP4 region as measured by bisulfite sequencing. (a) Scheme of the human IGF2 promoters (hP1-hP4, indicated by arrows) and CPG sites (in bold italics) in hP4. The locations of MON1-targeted sequence are underlined, and the putative TATA box is enclosed in the rectangle. The coding region of IGF2 is depicted in black boxes (GenBank accession no. C006408, 53861-53677). E1-E9 refers to exons 1-9. (b) DNA methylation profile in the hP4 region. Genomic DNA was treated with sodium bisulfite. The bottom strand was amplified with PCR and then sequenced using a sequencing kit. CpGs (5 and 6) were within the MON1 sequence. Note that almost all cytosines in hP4 are unmethylated in control cells and thus were converted into thymidines after treatment. The preservation of the $C$ residues indicates that they were methylated, demonstrating that MON1 treatment induces DNA methylation in the target region.

\section{Figure 5}

DNA methylation analysis of IGF2 by Southern blotting. (a) Location of Hpall (CCGG) sites and labeling probe. (b) Southern blotting. Lane 1 shows a 1-kb marker; lanes 2-4, PBS control DNA; and lanes 5-7, MON1-treated DNA. Genomic DNA was digested with Pvull and then either with Hpall sensitive to DNA methylation or by Mspl insensitive to DNA methylation. MON1 induced DNA methylation at the promoter site of IGF2, and thus the 1084-bp band was resistant to DNA methylation-sensitive Hpall (lanes 5 and 6 ) but not to DNA methylation-insensitive Mspl (lane 7). DNA from control cells (lanes 3 and 4) was completely digested by Hpall, indicating that control DNA was not methylated in that region.

oligonucleotide MON1. Since MON2 was unable to block IGF2 mRNA accumulation in vitro (Figure 3), it was used as a control oligonucleotide.

Untreated nude mice developed liver tumors soon after Hep 3B cell implantation. Four weeks after Hep 3B cell implantation, $\alpha$-fetoprotein (AFP), a tumor-derived serum marker, became detectable in the circulation. AFP levels increased dramatically thereafter, correlating with tumor growth (unpublished data). The beneficial effect of MON1 was reflected in the overall survival rate of the nude mice (Figure 7). In the first study, after 2 months of treatment, all of the saline-injected animals a

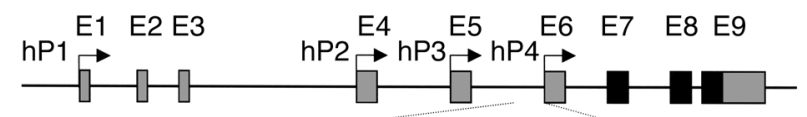

GGATGGTCCAGGGCTCC $\frac{C G G T T G G G G G T G C A G G A G A G A A A G A C T G G C T G G G}{1}$

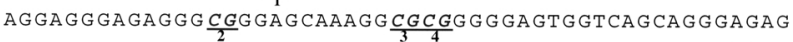

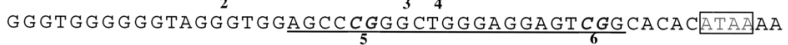
GCTGAGGCACTGACCAGCCTGCAAACTGG

b

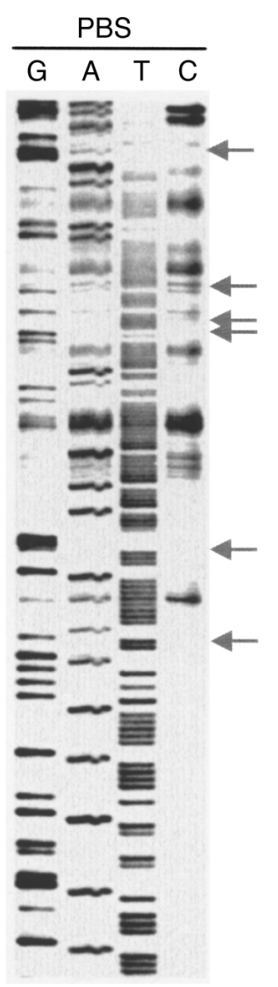

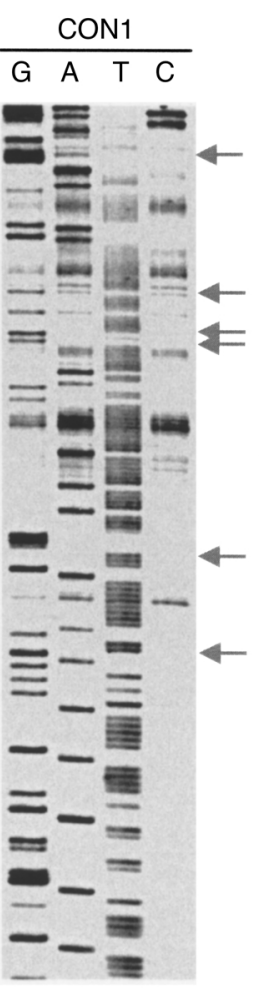




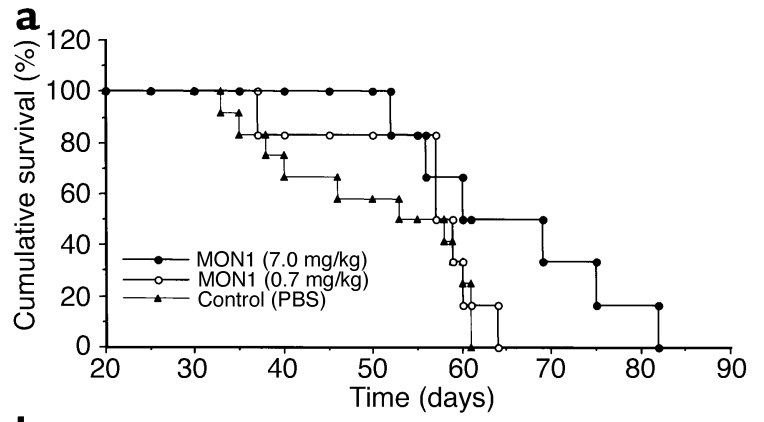

b

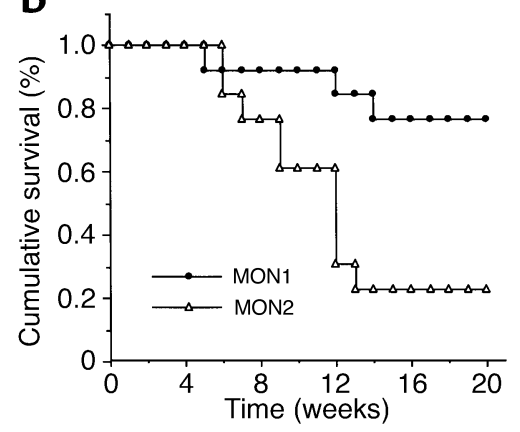

Figure 7

Cumulative survival rate of nude mice implanted with Hep 3B tumor cells. (a) In study 1, athymic nude mice were implanted with Hep 3B tumor cells $\left(10^{7}\right.$ cells). Ten days after tumor cell implantation, animals were randomly assigned into treatment groups and began to receive MON1 at doses of $0.7 \mathrm{mg} / \mathrm{kg}$ and $7 \mathrm{mg} / \mathrm{kg}$ and PBS control. Animals were dosed intravenously through the tail vein twice a week. At the end of the study, animals receiving MON1 treatment ( 7 $\mathrm{mg} / \mathrm{kg}$ ) had significantly prolonged survival in a clear dose-dependent manner $(P<0.05$ by the Mantel-Cox log-rank test).(b) In study 2 , nude mice were implanted with $10^{7} \mathrm{Hep} 3 \mathrm{~B}$ cells. Four weeks after implantation, animals were randomly assigned to receive MON2 (10 $\mathrm{mg} / \mathrm{kg}, n=13)$ or MON1 (10 mg/kg, $n=13)$. All treatments were administered through tail-vein injection twice a week. The MantelCox log-rank test shows a significantly prolonged survival in animals receiving MON1 treatment $(10 \mathrm{mg} / \mathrm{kg})$ as compared with those receiving MON2 treatment $(P<0.05)$.

had died, but only half of the mice treated with $7 \mathrm{mg} / \mathrm{kg}$ of MON1 had died by that time (Figure 7a). Animals injected with only $0.7 \mathrm{mg} / \mathrm{kg}$ of MON1 had a survival time similar to that of the saline-treated animals. To determine the specificity of the ability of MON1 to prolong survival in this model, mice carrying Hep 3B tumors were injected with MON1 or with MON2, the shortened methylated oligonucleotide that did not alter IGF2 mRNA abundance in the in vitro studies. After 20 weeks of treatment, mice treated with MON1 had a significantly improved survival time as compared with animals that received MON2 $(P<0.05)$, demonstrating that the lack of in vitro efficacy correlated with diminished in vivo antitumor effect (Figure 7b).

\section{Discussion}

DNA methylation has emerged as an important epigenetic modification that regulates gene transcription. In general, DNA methylation is correlated with gene repression. When a gene is hypermethylated, especially in its promoter region, transcription is usually diminished. Methylation of the cytosine in $\mathrm{CPG}$ dinucleotides has been shown to affect the expression of (1) genes that are expressed in a tissue-specific (22) or a development-specific manner $(26,27)$, (2) imprinted genes (28-30), (3) genes turned off by X-chromosome inactivation $(31,32),(4)$ some transgenes that are silenced and not transcribed (33-35), and (5) tumor suppressor genes in some neoplastic cells (36-38).

Gene expression from a transfected plasmid DNA can be efficiently suppressed by in vitro DNA methylation of CPG sites in the promoter region by using SssI methylase (39-41). When transfected into cells, the methylated plasmid DNA could not be transcribed by the endogenous RNA transcriptional machinery. Selective gene inactivation by DNA methylation has been demonstrated by using a methyltransferase/zinc-finger fusion protein model. The zinc-binding domain of the fusion protein binds to the targeted DNA sequence and guides CPG methylation by the SssI methylase domain at the specific site of the DNA sequence (42). In plants, doublestranded RNAs that are approximately 23 nucleotides in length can trigger de novo methylation of homologous DNA in the nucleus (43). The short RNAs are thought to act as guides for a methyltransferase complex that specifically methylates the complementary DNA sequence of silenced genes (44). It was assumed that during viral RNA-RNA replication, the RNA transcripts hybridize to their genomic counterparts and direct the sequence-specific DNA methylation $(45,46)$.

By analogy with this RNA-directed DNA methylation seen in plants, we have proposed a new method by which the expression of a given gene can be specifically blocked in mammalian cells. We have suggested that by synthesizing a short methylated sense oligonucleotide that is complementary to the sequence of the promoter region of a gene, we can induce hypermethylation of the promoter region of that gene and thus inhibition of gene transcription. We therefore designed a methylated oligonucleotide, MON1, that would bind to the promoter region of human IGF2 P4 and mouse Igf 2 P3.

Using hepatocyte model systems, we have shown that MON1 enters the nuclear compartment and inhibits IGF2 mRNA accumulation in normal and neoplastic hepatocytes. The unmethylated oligonucleotide ON1 had no inhibitory activity. As shown in Figures 5 and 6, MON1 treatment leads to increased methylation of IGF2 in the promoter $\mathrm{P} 4$ region.

DNA methylation as an epigenetic tag is usually heritable and stable in genomic imprinting. However, DNA methylation in nonimprinted genes, such as tumor suppressors, is always subject to dynamic change. MON1induced DNA methylation is an "artificial" epigenetic addition to IGF2 hP4. In our studies, we observed that the silenced IGF2 resumed its transcription two passages after termination of MON1 treatment (data not shown). Thus, the destiny of this "enforced" epigenetic mark by MON1 may replicate the demethylating fate of some $\mathrm{CPG}$ dinucleotides found in nonimprinted genes, 
suggesting that in the absence of repeated MON1 treatment, DNA demethylation may occur within the cell, leading to the loss of inhibition of the epigenetic mark and resumption of DNA transcription. Another likely explanation for the return of IGF2 transcription is the possibility that cells that no longer transcribe IGF2 are at a marked survival disadvantage as compared with those cells making abundant amounts of this mitogenic factor. Thus, those few cells which escape oligonucleotide-induced methylation silencing of IGF2 will grow at a greater rate than the affected cells and will ultimately become the predominant cell type.

Since IGF-II is a mitogenic peptide that has been shown to enhance tumor growth by autocrine and/or paracrine interactions, it is likely that inhibition of its synthesis would diminish tumor growth. In accordance with this conjecture, we created a xenotransplant model with athymic nude mice, similar to that described by $\mathrm{Bu}$ et al. (47). We found that mice that received the active oligonucleotide MON1 had a significantly greater survival rate than animals that received saline. In order to demonstrate the specificity of MON1, we also treated a group of animals with MON2, a methylated oligonucleotide that is three bases shorter and does not alter IGF2 levels in vitro. Mice receiving MON1 had a greater survival rate at 20 weeks than those mice that received MON2 (77\% vs. $21 \%, P<0.05$ by the log-rank test). All deaths were associated with visible tumor burden, and there were no observed acute, postinfusion events leading to death. Although we believe the desirable actions of this molecule are mediated through site-specific induction of methylation, we cannot rule out other ancillary effects, such as the methylation of other genes or decoy binding of transcription factors.

The development and growth of hepatic tumors in vivo is a complex process, and the increased expression and mitogenic activity of IGF-II, acting in an autocrine or paracrine fashion, is a key event (48). Others have shown that IGF-II may promote angiogenesis, thereby giving IGF2 an additional role in tumor growth (49). Using an antisense oligonucleotide directed against IGF2, Lin et al. were able to decrease proliferative activity in hepatoma cell lines, selectively decreasing IGF2 mRNA and protein (50). By suppressing IGF2 transcription and IGF2 production by MON1, we may intercede in this self-perpetuating cycle of growth and angiogenesis.

Antisense oligonucleotides to IGF2 mRNA have been shown to have suppressive effects on tumor cell growth. However, it is our belief that oncogene suppression at the transcriptional level is preferable to inhibition at the translational level, as transcriptional suppression requires far fewer copies of oligonucleotide per cell to be effective. Ideally, the induction of site-specific methylation can be accomplished by one copy of the methylated sense oligonucleotide per cell, whereas hundreds to thousands of antisense oligonucleotides per cell would be needed to bind and inactivate each mRNA and thereby reduce synthesis of the encoded protein. The lower dose afforded by the methylated-oligonucleotide strategy should decrease or abolish many of the side effects that have been seen in previous antisense animal and human trials $(51,52)$.

Previous work on methylation in tumors focused on altering methylation on a genome-wide basis (53). Although this technique may be helpful for studying the role of methylation in tumors, it is unlikely to be clinically useful. This is because genome-wide epigenetic alterations may lead to the relaxation of imprinting of growth-promoting genes. In humans, IGF2 is normally maternally imprinted in extrahepatic peripheral tissues, whereas in mice, it is imprinted in all tissues outside of the CNS. Administration of 5-azacytidine to mice leads to loss of Igf2 imprinting and aberrant imprinting of Igf2 (24). In contrast, our method has the potential to alter methylation in a gene-specific manner, leaving the remainder of methylated and imprinted genes unaltered. This specific epigenetic modification should be permanent and may remain intact in daughter cells after at least one cell division. Indeed, we observed in our in vitro studies that the inhibiting effect of MON1 on IGF2 expression could be maintained in Hep 3B cells for at least one more passage without further oligonucleotide treatment. In theory, any gene could be similarly targeted by a specific methylated oligonucleotide.

It has been shown that the unmethylated $\mathrm{CPG}$ motif located in an oligonucleotide sequence can cause Bcell proliferation and cytokine release $(54,55)$. This might be considered a confounding factor in tumorigenesis. However, Stein notes that methylation of the cytosine at $\mathrm{C} 5$, as we have done in our oligonucleotides, eliminates this effect (56).

In summary, we have shown that a modified oligonucleotide can alter the epigenome by inducing methylation of a previously unmethylated segment of DNA. This change in methylation correlates with downregulated expression of IGF2 mRNA. Once injected into the mouse with an implanted hepatocellular carcinoma, the methylated oligonucleotide preferentially accumulates in hepatocyte nuclei and leads to improved survival by suppressing the expression of the IGF2 gene.

\section{Acknowledgments}

This work was supported by the Medical Research Service of the Department of Veterans Affairs and NIH Grant DK36054.

\footnotetext{
1. Nakae, J., Kido, Y., and Accili, D. 2001. Distinct and overlapping functions of insulin and IGF-I receptors. Endocr. Rev. 22:818-835.

2. Cariani, E., et al. 1988. Differential expression of insulin-like growth factor II mRNA in human primary liver cancers, benign liver tumors, and liver cirrhosis. Cancer Res. 48:6844-6849.

3. Kim, S.O., Park, J.G., and Lee, Y.I. 1996. Increased expression of the insulin-like growth factor I (IGF-I) receptor gene in hepatocellular carcinoma cell lines: implications of IGF-I receptor gene activation by hepatitis B virus X gene product. Cancer Res. 56:3831-3836.

4. Pulford, D.J., Falls, J.G., Killian, J.K., and Jirtle, R.L. 1999. Polymorphisms, genomic imprinting and cancer susceptibility. Mutat. Res. 436:59-67.

5. Zhang, L., et al. 1997. Gene expression profiles in normal and cancer cells. Science. 276:1268-1272.
} 
6. Schirmacher, P., et al. 1992. Reactivation of insulin-like growth factor II during hepatocarcinogenesis in transgenic mice suggests a role in malignant growth. Cancer Res. 52:2549-2556.

7. Hu, J.F., et al. 1997. Repression of hepatitis B virus (HBV) transgene and HBV-induced liver injury by low protein diet. Oncogene. 15:2795-2801.

8. Su, Q., et al. 1994. Expression of insulin-like growth factor II in hepatitis B, cirrhosis and hepatocellular carcinoma: its relationship with hepatitis B virus antigen expression. Hepatology. 20:788-799.

9. Rogler, C.E., et al. 1994. Altered body composition and increased frequency of diverse malignancies in insulin-like growth factor-II transgenic mice. J. Biol. Chem. 269:13779-13784.

10. Christofori, G., Naik, P., and Hanahan, D. 1994. A second signal supplied by insulin-like growth factor II in oncogene-induced tumorigenesis. Nature. 369:414-418.

11. Haddad, R., and Held, W.A. 1997. Genomic imprinting and IGF-2 influence liver tumorigenesis and loss of heterozygosity in SV40 T antigen transgenic mice. Cancer Res. 57:4615-4623.

12. Christofori, G., Naik, P., and Hanahan, D. 1995. Deregulation of both imprinted and expressed alleles of the insulin- like growth factor 2 gene during beta-cell tumorigenesis. Nat. Genet. 10:196-201.

13. Gonzalgo, M.L., and Jones, P.A. 1997. Mutagenic and epigenetic effects of DNA methylation. Mutat. Res. 386:107-118

14. Cameron, E.E., Bachman, K.E., Myohanen, S., Herman, J.G., and Baylin, S.B. 1999. Synergy of demethylation and histone deacetylase inhibition in the re- expression of genes silenced in cancer. Nat. Genet. 21:103-107.

15. Hu, J.F., Vu, T.H., and Hoffman, A.R. 1996. Promoter-specific modulation of insulin-like growth factor II genomic imprinting by inhibitors of DNA methylation. J. Biol. Chem. 271:18253-18262.

16. Toulme, J.J., Di Primo, C., and Moreau, S. 2001. Modulation of RNA function by oligonucleotides recognizing RNA structure. Prog. Nucleic Acid Res. Mol. Biol. 69:1-46.

17.Szyf, M., and Detich, N. 2001. Regulation of the DNA methylation machinery and its role in cellular transformation. Prog. Nucleic Acid Res. Mol. Biol. 69:47-79.

18. Jones, P.L., et al. 1998. Methylated DNA and MeCP2 recruit histone deacetylase to repress transcription. Nat. Genet. 19:187-191.

19. Hu, J.F., Oruganti, H., Vu, T.H., and Hoffman, A.R. 1998. The role of histone acetylation in the allelic expression of the imprinted human insulin-like growth factor II gene. Biochem. Biophys. Res. Commun. 251:403-408.

20. Hu, J., Vu, T., and Hoffman, A. 1995. Differential biallelic activation of three insulin-like growth factor II promoters in the mouse central nervous system. Mol. Endocrinol. 9:628-636.

21. Li, T., et al. 2002. An imprinted PEG1/MEST antisense expressed predominantly in human testis and in mature spermatozoa. J. Biol. Chem. 277:13518-13527.

22. Hu, J.F., Oruganti, H., Vu, T.H., and Hoffman, A.R. 1998. Tissue-specific imprinting of the mouse insulin-like growth factor II receptor gene correlates with differential allele-specific DNA methylation. Mol. Endocrinol. 12:220-232.

23. Frommer, M., et al. 1992. A genomic sequencing protocol that yields a positive display of 5-methylcytosine residues in individual DNA strands. Proc. Natl. Acad. Sci. USA. 89:1827-1831.

24. Hu, J.F., Nguyen, P.H., Pham, N.V., Vu, T.H., and Hoffman, A.R. 1997 Modulation of IGF-2 genomic imprinting in mice induced by 5 -azacytidine, an inhibitor of DNA methylation. Mol. Endocrinol. 11:1891-1898.

25. Vu, T.H., et al. 2000. Symmetric and asymmetric DNA methylation in the human IGF-2-H19 imprinted region. Genomics. 64:132-143.

26. Lubbert, M., Brugger, W., Mertelsmann, R., and Kanz, L. 1996. Developmental regulation of myeloid gene expression and demethylation during ex vivo culture of peripheral blood progenitor cells. Blood. 87:447-455.

27. Stoger, R., et al. 1993. Maternal-specific methylation of the imprinted mouse IGF-2r locus identifies the expressed locus as carrying the imprinting signal. Cell. 73:61-71.

28. Hark, A.T., et al. 2000. CTCF mediates methylation-sensitive enhancerblocking activity at the H19/IGF-2 locus. Nature. 405:486-489.

29. Bell, A.C., and Felsenfeld, G. 2000. Methylation of a CTCF-dependent boundary controls imprinted expression of the IGF-2 gene. Nature. 405:482-485.
30. Nakagawa, H., et al. 2001. Loss of imprinting of the insulin-like growth factor II gene occurs by biallelic methylation in a core region of H19associated CTCF-binding sites in colorectal cancer. Proc. Natl. Acad. Sci. USA. 98:591-596.

31. Panning, B., and Jaenisch, R. 1996. DNA hypomethylation can activate Xist expression and silence X-linked genes. Genes Dev. 10:1991-2002.

32. Beard, C., Li, E., and Jaenisch, R. 1995. Loss of methylation activates Xist in somatic but not in embryonic cells. Genes Dev. 9:2325-2334.

33. Schweizer, J., Valenza-Schaerly, P., Goret, F., and Pourcel, C. 1998. Control of expression and methylation of a hepatitis B virus transgene by strain-specific modifiers. DNA Cell Biol. 17:427-435.

34. Siegfried, Z., et al. 1999. DNA methylation represses transcription in vivo. Nat. Genet. 22:203-206.

35. Scrable, H., and Stambrook, P.J. 1997. Activation of the lac repressor in the transgenic mouse. Genetics. 147:297-304.

36. Esteller, M., Corn, P.G., Baylin, S.B., and Herman, J.G. 2001. A gene hypermethylation profile of human cancer. Cancer Res. 61:3225-3229.

37. Tycko, B. 2000. Epigenetic gene silencing in cancer. J. Clin. Invest. 105:401-407.

38. Jones, P.A. 2001. Cancer. Death and methylation. Nature. 409:141-144.

39. Song, S.H., et al. 2001. Transcriptional silencing of cyclooxygenase- 2 by hyper-methylation of the 5' $\mathrm{CPG}$ island in human gastric carcinoma cells. Cancer Res. 61:4628-4635.

40. Borde-Chiche, P., et al. 2001. Regulation of transcription of the glutathione S-transferase P1 gene by methylation of the minimal promoter in human leukemia cells. Biochem. Pharmacol. 61:605-612.

41. Levine, A., Cantoni, G.L., and Razin, A. 1991. Inhibition of promoter activity by methylation: possible involvement of protein mediators. Proc. Natl. Acad. Sci. USA. 88:6515-6518.

42. Xu, G.L., and Bestor, T.H. 1997. Cytosine methylation targeted to predetermined sequences. Nat. Genet. 17:376-378.

43. Wassenegger, M. 2000. RNA-directed DNA methylation. Plant Mol. Biol. 43:203-220.

44. Pelissier, T., and Wassenegger, M. 2000. A DNA target of $30 \mathrm{bp}$ is sufficient for RNA-directed DNA methylation. RNA. 6:55-65.

45. Wassenegger, M., Heimes, S., Riedel, L., and Sanger, H.L. 1994. RNAdirected de novo methylation of genomic sequences in plants. Cell. 76:567-576.

46. Wassenegger, M., and Pelissier, T. 1998. A model for RNA-mediated gene silencing in higher plants. Plant Mol. Biol. 37:349-362.

47. Bu, W., et al. 1998. Effects of matrix metalloproteinase inhibitor BB-94 on liver cancer growth and metastasis in a patient-like orthotopic model LCI-D20. Hepatogastroenterology. 45:1056-1061.

48. Scharf, J.G., Dombrowski, F., and Ramadori, G. 2001. The IGF axis and hepatocarcinogenesis. Mol. Pathol. 54:138-144.

49. Lee, O.H., et al. 2000. Identification of angiogenic properties of insulinlike growth factor II in in vitro angiogenesis models. Br. J. Cancer. 82:385-391.

50. Lin, S.B., et al. 1997. Antisense oligodeoxynucleotides of IGF-2 selectively inhibit growth of human hepatoma cells overproducing IGF-2. J. Biochem. (Tokyo). 122:717-722.

51. Henry, S.P., Monteith, D., Bennett, F., and Levin, A.A. 1997. Toxicological and pharmacokinetic properties of chemically modified antisense oligonucleotide inhibitors of PKC-alpha and C-raf kinase. Anticancer Drug Des. 12:409-420.

52. Rudin, C.M., et al. 2001. Phase I Trial of ISIS 5132, an antisense oligonucleotide inhibitor of c- raf-1, administered by 24-hour weekly infusion to patients with advanced cancer. Clin. Cancer Res. 7:1214-1220.

53. Karpf, A.R., et al. 1999. Inhibition of DNA methyltransferase stimulates the expression of signal transducer and activator of transcription 1, 2, and 3 genes in colon tumor cells. Proc. Natl. Acad. Sci. USA. 96:14007-14012.

54. Sparwasser, T., Hultner, L., Koch, E.S., Luz, A., Lipford, G.B., and Wagner, H. 1999. Immunostimulatory CpG-oligodeoxynucleotides cause extramedullary murine hemopoiesis. J. Immunol. 162:2368-2374.

55. Krieg, A.M., et al. 1995. CpG motifs in bacterial DNA trigger direct B-cell activation. Nature. 374:546-549.

56. Stein, C.A. 1999. Two problems in antisense biotechnology: in vitro delivery and the design of antisense experiments. Biochim. Biophys. Acta. 1489:45-52. 